\title{
An exploration of factors related to healthy weight maintenance amongst staff at an Irish University
}

\author{
Ann Reilly ${ }^{1}$, Barbara Mawn ${ }^{2}$, Anthony Staines ${ }^{1}$, Sarah Browne ${ }^{1}$, Davide Susta ${ }^{1}$ \\ and Mary Rose Sweeney ${ }^{1}$ \\ ${ }^{1}$ NURISH, Centre for Preventive Medicine, School of Nursing \& Human Sciences, DCU and ${ }^{2}$ University of Massachusetts \\ Lowell, USA
}

Overweight and obesity have increased dramatically in the past 20 years in Ireland with almost two thirds of the population now overweight ${ }^{(1)}$. According to a recent report, Ireland now ranks as one of the most overweight/obese nations in the EU ${ }^{(2)}$. In Ireland, it has been estimated that the economic impact of obesity is around $€ 1.6$ billion annually ${ }^{(3)}$. Much research has focused on achieving and maintaining weight loss, whereas no previous research has specifically studied the population who consistently successfully maintain their weight within 'normal ranges' (i.e. body mass index (BMI) from 20-25) over their lifetimes. With one in three people in Ireland maintaining a healthy weight, there is much to learn from this 'normal weight' group.

The aim of this research study was to examine the factors that contribute positively and negatively to one's ability to maintain a healthy body weight.

Staff across all departments and all sectors (Support, Academic, Administrative and Technical) in an Irish University were invited by email to take part in this research study. Focus groups were conducted as outlined below.

\footnotetext{
Focus group 1: Staff who actively maintain their weight within a healthy range $(n=7)$

Focus group 2: Staff who have lost over 14lbs (1 stone) in weight and managed to maintain it for over one year $(n-4)$

Focus group 3: Staff whose BMI or weight is considered overweight/obese who have not lost weight in the past year $(n=6)$

The focus groups took approximately 1 hour. The focus groups were transcribed verbatim and analysed into themes.
}

This research indicates that there are three broad elements to successful weight loss and weight maintenance:

Socio-cultural factors: including beliefs, community engagement, family and peer support.

Cognitive and emotional control: will power, persistence and perseverance.

Individualised strategies: planning, prioritising, portion control, calorie restriction and rowing back.

Our results shed light on the interplay of various factors involved in healthy weight maintenance and may also have implications for targeted interventions.

1. Irish Universities Nutrition Alliance (2011) National Adult Nutrition Survey Summary Report. http://www.iuna.net/wp-content/uploads/2010/12/ National-Adult-Nutrition-Survey-Summary-Report-March-2011.pdf.

2. HSE.ie (2009) Health status of the population of Ireland. http://www.hse.ie/eng/services/Publications/HealthProtection/Public_Health_/Health_ Status of the Population_of Ireland_2008.html.

3. Safefood.eu (2012) The cost of overweight and obesity on the Island of Ireland Executive Summary. http://www.safefood.eu/SafeFood/media/ SafeFoodLibrary/Documents/Publications/Research\%20Reports/Final-Exec-Summary-The-Economic-Cost-of-Obesity.pdf. 